\title{
Gravitational and matter-wave spectroscopy of atomic hydrogen at ultra-low energies
}

\author{
Sergey Vasiliev $^{1}$ (D) . Janne Ahokas ${ }^{1}$. Jarno Järvinen ${ }^{1} \cdot$ Valery Nesvizhevsky $^{2}$. \\ Alexei Voronin ${ }^{3}$. François $\mathrm{Nez}^{4}$. Serge Reynaud ${ }^{4}$
}

Published online: 4 February 2018

(C) The Author(s) 2019

\begin{abstract}
We propose experiments with atomic hydrogen gas at ultra-low temperatures $T<100 \mu \mathrm{K}$ when the thermal energy of atoms is comparable with the changes of their potential energy in the Earth gravity field. At these conditions we suggest implementing a gravitational spectroscopy for studies of quantum properties of ultra-cold atomic hydrogen and its interactions with matter and gravity, similar to experiments with ultra-cold neutrons (Nesvizhevsky et al. Nature 415, 297 2002). A magnetic trap used for reaching the Bose-Einstein Condensation (Fried et al. Phys. Rev. Lett. 81, 3811 1998) can be used for cooling a large number of H atoms below $1 \mathrm{mK}$. Evaporative cooling over the trap barrier allows effective cooling of the vertical degree of freedom of the trapped atoms. Releasing these ultra-slow atoms from the trap onto the cold surface of superfluid helium will allow studies of quantum bounces and stationary gravitational states of $\mathrm{H}$ atoms in the potential well created by this surface and the field of Earth gravity. Experimental study of properties of gravitational quantum states of hydrogen and quantum reflection of ultracold hydrogen from surface would be of major importance for designing similar experiments with antihydrogen, which are currently prepared in CERN.
\end{abstract}

Keywords Atomic hydrogen · BEC - Quantum reflection

This article is part of the Topical Collection on Proceedings of the 13th International Conference on Low Energy Antiproton Physics (LEAP 2018) Paris, France, 12-16 March 2018

Edited by Paul Indelicato, Dirk van der Werf and Yves Sacquin

\footnotetext{
Sergey Vasiliev
}

servas@utu.fi

1 Department of Physics and Astronomy, University of Turku, Vesilinnantie 5, 20014 Turku, Finland

2 Insitut Laue-Langevin (ILL), 71 avenue des Matryrs, 38042, Grenoble, France

3 P. N. Lebedev Physical Institute, 53 Leninsky prospect, 117924, Moscow, Russia

4 Laboratoire Kastler Brossel, Sorbonne Université, CNRS, ENS-Université PSL, Collége de France, 75252, Paris, France 


\section{Introduction}

The quantum properties of matter are usually studied in the microscopic world and under the extreme conditions of ultralow temperatures, or slow atomic motion. Perhaps the most known quantum phenomenon observed under these conditions is the Bose-Einstein Condensation (BEC). The appearance of the condensate is associated with a drastic change of macroscopic properties of the gaseous cloud. Most spectacular is the phenomenon of superfluidity and presence of quantized vortices. Nowadays BECs of alkali atoms are studied in many laboratories, owing to a remarkable progress in this field since the experiments of E. Cornell, K. Wieman [3], and W. Ketterle [4] in 1995. Most of the experimental methods used to create condensates of alkali vapours were originally invented and tested on hydrogen. A study of properties of $\mathrm{H}$ was always of prime importance because they can be calculated from the first principles. Therefore, as the element number one, $\mathrm{H}$ has often served as a test probe of the fundamental laws of physics. BEC was achieved in the gas of atomic hydrogen by the group of D. Kleppner at MIT [2], and in the two-dimensional H gas at the University of Turku [5] in 1998.

Unification of quantum mechanics with the theory of gravity remains one of the unresolved problems in the modern theories, and experimental investigation of the gravitational properties of quantum mechanical objects, such as light neutral atoms or elementary particles, is of great importance. Experiments with gravitational states of ultracold neutrons opened a new way for studies of fundamental properties of matter and gravity [1], and similar experiments are suggested for atomic hydrogen and antihydrogen [6].

In this paper, we propose to implement a gravitational spectroscopy for studies of quantum properties of ultra-cold atomic hydrogen and its interactions with matter and gravity. We are going to build a magnetic trap for cooling a large number of $\mathrm{H}$ atoms below $1 \mathrm{mK}$. We will release ultra-slow atoms from the trap onto the cold surface of superfluid helium. Owing to the weakest interaction potential such surface provides nearly perfect reflecting conditions for low-energy atoms. This will allow studies of quantum bounces and stationary gravitational states of $\mathrm{H}$ atoms in the potential well created by this surface and the field of Earth gravity. The methods and ideas of this project will be useful for making similar experiments with antihydrogen utilizing a new source and trap for anti-H currently prepared in CERN [7-10].

\section{Background}

Research work on cooling neutral gases was at first concentrated on reaching BEC for the lightest possible atom - atomic hydrogen. Atomic hydrogen gas is metastable against recombination into molecules, and in order to prevent this one should polarize its electron spin. This can be done by the brute force, applying high magnetic fields at low temperatures, when the thermal energy becomes much smaller than the Zeeman interaction with the magnetic field. Technically reaching these conditions was fairly easy already in the middle of the previous century, routine dilution refrigerator could reach temperatures below $0.1 \mathrm{~K}$, and simple superconducting magnet could create strong enough magnetic fields of several T. The atoms with the electron spins directed opposite to the polarising magnetic field (high field seekers) collect in the region of strong magnetic field and can be accumulated there at high density. However, another problem arose: Van der Waals interaction with any physical wall is so strong that cooling a gas of $\mathrm{H}$ inside a container leads to its condensation (adsorption) on the walls with subsequent depolarization of electron spin and recombination 
already at temperatures of the order of $10 \mathrm{~K}$. At this stage, researchers came across with the problem of finding material with the weakest possible interaction with $\mathrm{H}$ atoms. It turned out that such material is liquid helium, and owing to its superfluidity one may cover all surface of the container by a superfluid helium film. This led the first stabilization of cold atomic hydrogen gas in the pioneering experiments of I. Silvera and J. Walraven at the University of Amsterdam in 1979 [11] at the temperature of $270 \mathrm{mK}$ and density of the order of $n \sim 10^{14} \mathrm{~cm}^{-3}$.

Subsequent experiments confirmed that $\mathrm{H}$ has relatively weak attractive interaction with helium surface. The shallow potential well supports only one bound state with an adsorption energy of $\sim 1 \mathrm{~K}$. Improvements in the experimental technique allowed compression of $\mathrm{H}$ gas to densities of the order of $5 \times 10^{18} \mathrm{~cm}^{-3}$, but this was still quite far from conditions required for BEC. At so high densities, phase space compression was limited by the threebody recombination. Adsorption on the walls and subsequent surface recombination limited cooling to BEC at low densities. Thus, by the middle of 1980, it became clear that one cannot reach quantum degeneracy in three-dimensional $\mathrm{H}$ gas confined by the material walls.

In 1986 Harald Hess suggested another way for reaching BEC in atomic hydrogen [12]. He proposed to build a magnetic trap where the wall-free confinement is provided by magnetic forces. It is not possible to create a magnetic field configuration with magnetic field maximum in the current-free space, but a local minimum can be created in a configuration of currents known as the Ioffe-Pritchard trap. The atoms with the direction of the electron spin along magnetic field are attracted to the minimum of the magnetic field. These, so-called low field seekers, can be trapped in magnetic traps. Hess suggested a method of cooling the trapped atoms by evaporation of the hottest atoms over the trap barrier with subsequent thermalization of remaining gas due to interatomic collisions. This proposal opened a new way for cooling the atoms, and experiments were immediately initiated by D. Kleppner and T. Greytak in MIT and by J. Walraven in the University of Amsterdam. Their efforts led to the successful BEC experiment with $\mathrm{H}$ in 1998 [2], which was however three years later than it was done for the alkali atoms of ${ }^{87} \mathrm{Rb}[3,4]$.

Interaction of $\mathrm{H}$ atoms with helium surface and adsorbed two-dimensional phase of $\mathrm{H}$ concerns rather interesting physics and deserved special studies. The surface of liquid helium is the best possible surface for experiments at ultralow energies [13]. It is extremely pure, ideally flat and atomically smooth. The surface excitations - ripplons completely vanish at $100 \mathrm{mK}$ which greatly simplifies analysis. Calculation of the H-liquid He interaction potential seems to be fairly simple since we deal with the simplest and lightest atoms. However, here we again come to the situation that our intuitive prediction based on the classical treatment gives a wrong result. While a classical analysis reveals that the sticking probability $s$ in the collisions of low-energy atoms approaches unity at low temperatures, the quantum treatment predicts that it should follow universal $\sqrt{T}$ dependence and approach zero at $T=0$. The reason for such behaviour at low temperatures is the absence of surface excitations, which ensure the energy and momentum conservation after adsorption of the atom in the surface potential well.

The quantum behaviour of the reflection was observed in experiments of Amsterdam group as the enhanced capillary flow of $\mathrm{H}$ atoms [14] and focusing of the atomic beam with a concave mirror [15]. In this work, a linear decrease of the sticking probability with temperature was observed which contradicted the theoretical predictions. In later experiments of MIT group [16], the atoms were cooled to much lower temperatures in the magnetic trap and released on to the surface of superfluid helium. Expected $\sqrt{T}$ dependence was 
confirmed in these experiments, but the value of the sticking probability was substantially higher than those measured by the Amsterdam group. A possible source of error in the MIT work is the poor accuracy of the film thickness measurement. It seems that the influence of metallic substrate was not completely eliminated. We expect that the sticking probability of $\mathrm{H}$ will approach $\mathrm{s}=0.001$ at $100 \mu \mathrm{K}$ for collisions with really thick films or bulk helium. This vanishing sticking probability at low energies is very important for the proposed experiments.

\section{Proposed experiments}

Cooling $\mathrm{H}$ atoms to lowest possible energies is a somewhat different task from reaching BEC conditions. In the latter task, one should compress the gas in the phase space, keeping high density of atoms at lowest possible temperature. Collisions between the atoms should establish thermal equilibrium which is continuously disturbed by the evaporative cooling, when the hottest atoms are kicked out from the trap. Owing to the very small size of $\mathrm{H}$, the collision cross section is very small, and to provide high enough equilibration rate one has to keep the gas at sufficiently high density. In the MIT experiments [2] densities approaching $5 \times 10^{15}$ were reached that is an order of magnitude larger than in typical BEC experiments with other alkali vapours. Even at that high densities it turned out to be impossible to reach full thermal equilibrium, and the axial and radial motion of the atoms were essentially decoupled. Another serious problem arose at high densities from the two-body collisions of atoms, which lead to the spin flips caused by the dipolar interaction of the colliding atoms. The atoms with the flipped spin are immediately pushed out from the trap. This process of dipolar relaxation occurs mainly near the bottom of the trap where the atoms are having lowest kinetic energy, and leads to effective heating of the gas.

The rate of dipolar relaxation may be strongly reduced at lower densities, since this is a two-body process and its rate if proportional to $n^{2}$. At densities below $10^{13} \mathrm{~cm}^{-3}$ lifetime of the sample in the MIT trap, limited by the dipolar relaxation, would be several hours, and this process will not lead to a significant heating of the gas. The thermalization rate of the gas is proportional to the collision rate and also decreases at lower densities, which makes more difficult reaching thermal equilibrium in the gas. The evaporative cooling becomes slow and inefficient, leading to decoupling of radial and axial motion of the atoms and strong departure from thermal equilibrium $[17,18]$. However, equilibrium is not necessary for the gravitational spectroscopy experiments, and therefore we will follow a different pathway in the phase space than was used in the MIT experiment. We will be aiming at slowing axial (vertical) motion of the atoms to energies comparable with their potential in the field of gravity.

A second feature of the quantum reflections which we are going to use is the vanishing sticking probability at low energies. Releasing low energy atoms into a container with helium covered walls will lead to an enhanced adsorption of the highest energy atoms, and lead to an "absorptive" cooling, in this sense similar to the evaporation over the magnetic trap barrier. What is important is that this process does not require collisions of the atoms with each other, and the dipolar relaxation is ruled out of the game.

These two basic features can be effectively used in two types of experiments: 1) with a beam of low energy atoms, similar to the experiments with ultra-cold neutrons [1], and 2) trapping the ultra-low energy atoms in a static trap having bulk superfluid helium at the bottom. We describe both proposed experiments in more details in the following sections. 


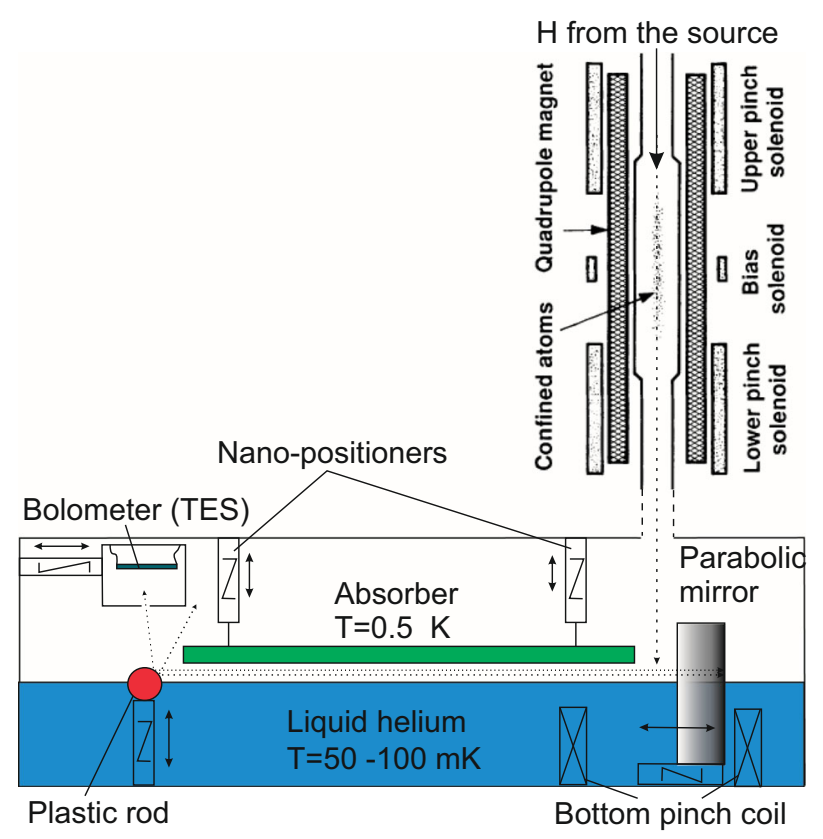

Fig. 1 Schematic diagram of the experimental setup for the beam type experiment to detect gravitational states of ultra-cold $\mathrm{H}$ on liquid helium surface

In both cases, we suggest to use a magnetic trap of the Ioffe-Pritchard type (IPT), similar to that proposed by Hess [12] and used by the MIT group [18, 19]. The trap axis is aligned vertically, as depicted in Fig. 1. Increasing the volume of the trap and changing the radial trapping geometry from quadrupole to octupole will allow trapping at least an order of magnitude larger number of atoms than in MIT work. One may load the trap with $N>$ $10^{15}$ atoms at temperature of $T \sim 50 \mathrm{mK}$. Then, using a forced evaporative cooling along vertical axis, the temperature of the gas is lowered to $T=1 \mathrm{mK}$ with $N \sim 3 \times 10^{13}$ and density of the gas $n \sim 10^{13} \mathrm{~cm}^{-3}$. Using lower densities on the cooling path helps saving number of atoms. At this conditions the atoms are still in thermal equilibrium with average velocity of $\approx 5 \mathrm{~m} / \mathrm{s}$. Continuing decrease of the axial barrier leads to decoupling of axial and radial motion $[17,18]$, rate of the gas thermalization drops and evaporative cooling becomes inefficient. Further decrease of the vertical velocity $v_{z}$ may be done by truncation in the velocity space, quickly ramping down the axial barrier and removing atoms with energies larger than its final height. A decrease of one velocity component by the factor of 30 will require a reduction of the number of atoms by same number. Thus we will arrive to the trapped number of $\sim 10^{12}$ atoms of $\mathrm{H}$ gas at density of $10^{11}-10^{12} \mathrm{~cm}^{-3}$, with the axial velocity $\sim 15 \mathrm{~cm} / \mathrm{s}$ and its radial component of $\sim 5 \mathrm{~m} / \mathrm{s}$.

\subsection{Beam type}

A scheme of the proposed experiment is shown in Fig. 1. At the bottom of the trap, we suggest to arrange a chamber with the free surface of liquid helium and an absorber plate hanging above it with the variable distance of 10-100 $\mu \mathrm{m}$ to the helium surface. The gap between the adsorbed and helium surfaces is adjusted with cryogenic nanopositioners [20]. 
The absorber is hanged with thin superconducting wires, which provide thermal insulation, allow heating it to $0.5 \mathrm{~K}$, and evaporating helium film from it. The lower surface of absorber may be covered with carbon-based paint to increase adsorption properties of $\mathrm{H}$. At the bottom of the spectrometer chamber around the axis of the trap, one more pinch coil is arranged with nearly same parameters as the lower pinch solenoid of the trap. Running current of opposite direction in these two lower pinch coils will create another trapping configuration with the region of zero field which can be adjusted in the vertical direction by changing currents in the coils. Manipulating with these currents one can transfer the cloud of cold atoms down to the surface of liquid helium. One cannot simply drop the cloud few $\mathrm{cm}$ down by switching off the lower pinch field. The atoms would gain energy several times larger than what we ultimately reach for their vertical velocities. After bringing the cloud to the surface all magnetic fields are reduced to zero. The rate of the field change will define the flux of the atoms into the gap below absorber, and finally to the detector. Release of atoms cannot be very fast because of the eddy current heating, but we think that within a minimum time of $\sim 10 \mathrm{sec}$ this can be done. Fortunately, magnetic field strength is rather small $\sim 10 \mathrm{G}$ in the end of the evaporative cooling, and the rate of the field ramp $\sim 1$ G/s, two orders of magnitudes smaller than in MIT experiments, should not be a problem. The cloud will expand in the radial direction and the atoms will start to collide with the surface of parabolic mirror arranged around the axis of the traps. The position of the mirror focus is adjusted with nano-positioner to match the cloud center, so that the atoms moving in radial direction will be reflected in the form of a parallel beam. The cloud will expand in the radial direction and the atoms will start colliding with the surface of a parabolic mirror arranged around the axis of the traps. The position of the mirror focus is adjusted with nanopositioner to match the cloud center, so that the atoms moving in radial direction will be reflected in the form of a parallel beam.

The absorber will make a further selection of the atoms with smallest vertical velocities. The atoms having energy larger than the gravitational energy on the gap height will be captured on it's surface. The atoms passing between the surface of helium and absorber will settle in the gravitational states and the transmission of the beam may be measured as a function of the gap height, as it was done in the experiments with ultra-cold neutrons at ILL [1]. In order to increase the spatial resolution of the gravitational states, we propose to use the method realized by Ishikawa in experiments with UCN at ILL [21, 22]. The cylindrical rod is placed at the end of the atomic beam passing through the gap. With the full specular reflection of incident particles, it works as a convex lens, and the spatial distribution of the atomic beam is effectively magnified by the factor of 25 . The atoms reflected from the rod reach the detector, which will provide a measurement of the beam intensity as a function of the position in the horizontal plane. Clearly, in such experiment, most of the atoms initially collected in the trap will be lost and will not pass through the gap. Gravitational states will be resolved by varying the height of the gap within the range 10-100 $\mu \mathrm{m}(\sim 15$ lowest states [1]), which implies truncation in the vertical velocity by the factor of 3-10 and corresponding loss of atoms. The number of atoms entering the slot in the box with the bolometer will be further reduced by the factor $10^{2}-10^{3}$ defined by the desired resolution and collection angle of the detection system dependent of the final geometry. Considering other possible losses (sticking and scattering of the atoms, imperfect focusing) we make a conservative estimate of the total loss as 4-5 orders of magnitude from the number initially prepared in the IPT. However, even after that one will get $10^{7}-10^{8}$ atoms, which can be released during adjustable time. Sensitivity of the bolometer to detect recombination of $\sim 10^{6}$ atoms $/ \mathrm{s}$ demonstrated in MIT work [18, 19], seems to be good enough for detection of the flux of 


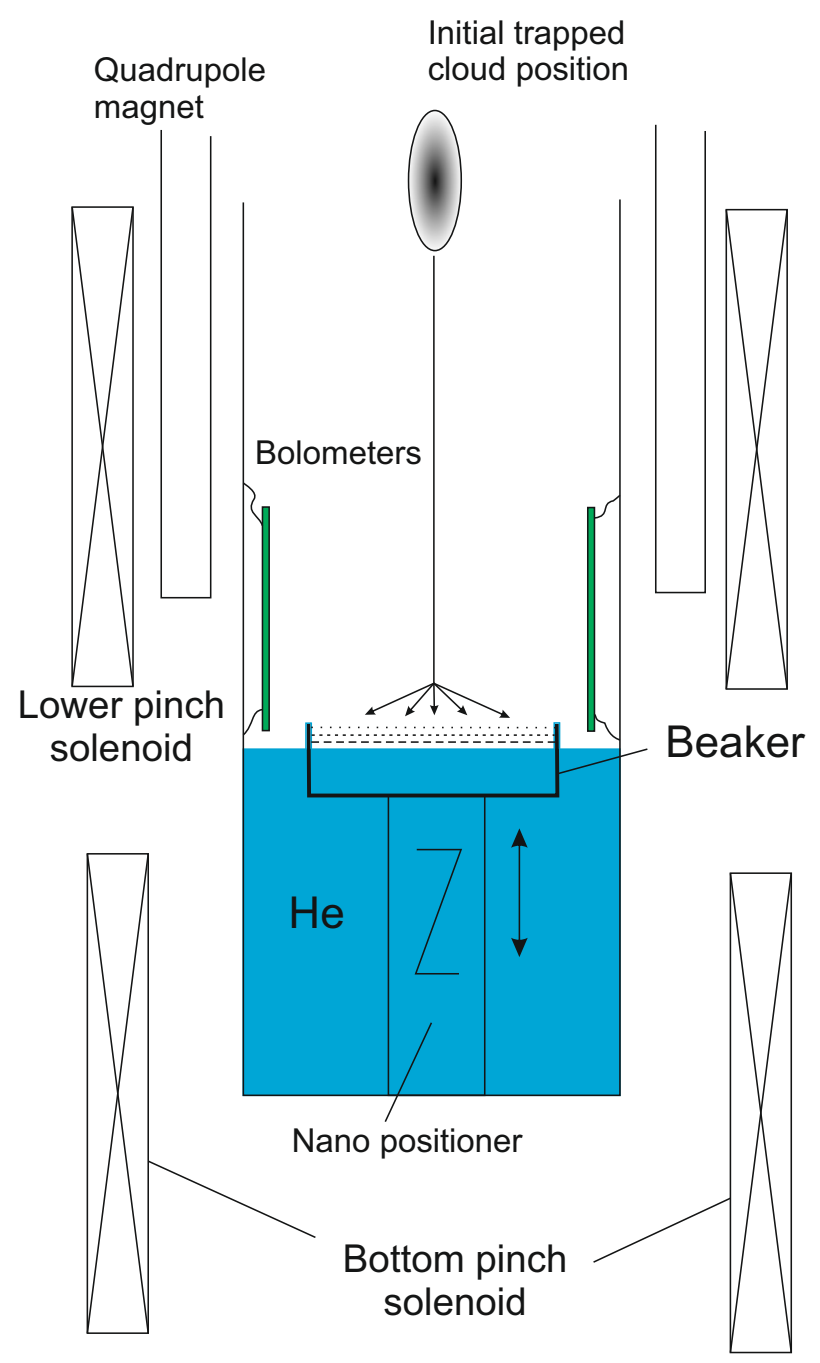

Fig. 2 Scheme of the stationary trap for experiments with fravitational states of hydrogen atoms

atoms passing through the gap and entering the gap of the box with the detector if they are released from the trap within several tens of seconds.

\subsection{Static trap}

A scheme of this experiment is shown in Fig. 2. The same trapping and cooling scenario will be used to obtain initial cloud of atoms. Similar to the beam experiment, one more pinch coil is arranged below the main Ioffe-Pritchard trap. After the initial stage of trapping and evaporative cooling, the atomic cloud with the starting parameters as described above is slowly transferred into a cylindrical beaker or piece of a glass tube with the walls sticking slightly above the surface of helium. In the beginning, the height of these physical walls 
will be $2-3 \mathrm{~cm}$, several times larger than the kinetic energy of atoms in the vertical direction. The gas will occupy the lower part of the beaker. Then, evaporative cooling above the wall will start to reduce the vertical velocity of the atoms. The cooling rate will be adjusted by lowering the beaker into the bulk helium. Finally, at the height of $<0.1 \mathrm{~mm}$ the flux of escaping atoms will depend on the proximity of the gravitational states. The changes in the flux of atoms escaping from such a trap will be detected by a bolometer attached to the cylindrical wall of the sample cell. In addition to the escape above the trap wall, the atoms will be lost due to inelastic collisions with the surface of liquid helium. We expect that this will be the main loss mechanism. However, since the sticking probability increases for larger velocities, this will remove most energetic atoms, and will lead to an effective cooling of the horizontal component. We estimate that at the horizontal velocity of $4 \mathrm{~m} / \mathrm{s}$ and $4 \mathrm{~cm}$ diameter of the beaker the mean flight time is $10^{-2} \mathrm{~s}$. With the sticking probability of $10^{-2}$ at $\mathrm{T}=1 \mathrm{mK}$ [16], the decay time of such hot atoms would be about $1 \mathrm{~s}$, if the reflection is specular enough. The decay will slow down as the high-energy atoms will disappear from the gas. Non-specular collisions with the walls and with the meniscus of helium near the trap bottom will lead to a mixing of all velocity components. To estimate the final number of atoms distributed in 5-10 lowest gravitational states in the trap of $\sim 0.1 \mathrm{~mm}$ height we assume that their energies will be cut above the height of the gravitational potential barrier $\sim 0.1 \mu \mathrm{K}$ corresponding to the thermal velocity of $v \sim 5 \mathrm{~cm} / \mathrm{s}$. This will require factor of 3 reduction in vertical and factors of 100 in both horizontal components, leading in total to a reduction of $3 .^{4}$ in number of atoms from initially prepared in IPT. Finally we may expect $3 \cdot 10^{7}$ atoms with thermal energies of $0.1 \mu \mathrm{K}$. The rate of the sticking collisions with the walls and bottom of the beaker can be estimated as $d N / d t \sim n v A s / 4 \approx 2 \cdot 10^{5} \mathrm{~s}^{-1}$, where $n \approx 4 \cdot 10^{8} \mathrm{~cm}^{-3}$ is the gas density, $A \approx 13 \mathrm{~cm}^{2}$ is the total area of trap (beaker) surface, $s \sim 3 \cdot 10^{-5}$ is the sticking probability at $0.1 \mu \mathrm{K}$ (we assume a square-root reduction of $s$ with temperature [19]). The lifetime of such ensemble of $N \sim 3 \cdot 10^{7}$ atoms will be of the order of several minutes. The energy levels will be well resolved and transitions between them can be excited via vibrations of the beaker by the piezo positioner. Exciting the transitions into the levels above the trap wall will lead to increased recombination rate, which can be analyzed using a bolometer. It is very important to have the initial temperature of the gas cloud before its transfer into the gravitational trap as low as possible.

\subsection{Diagnostic tools}

At the first stage of experiments in MIT, the $\mathrm{H}$ atoms were detected by the calorimetrical method. A sensitive carbon bolometer was used to measure heat liberated in atomic recombination. The sensitivity of their device was equivalent to $10^{6}$ atoms/s. Much more sensitive bolometers were developed since that time. Most sensitive are so-called Transition Edge Sensor (TES) [23]. They utilize steep dependence of the resistance of metals at the edge of the superconductor/normal transition. SQUIDs are typically used for the measurement of the resistance change. TES are commonly used in radio astronomy. They reach sensitivity sufficient for detection of a single infrared photon. The arrays of such sensors are developed, which can measure the spatial distribution of the energy flux, like a CCD of a camera. We suggest to place TES inside a small chamber with a slit for incident atoms. The TES will be weakly coupled to the chamber wall and heated slightly above ambient temperature to evaporate helium film from its surface. All incident atoms will adsorb on TES surface and recombine. We expect that such detector will be able to detect atomic fluxes of the 
order of $10^{2}-10^{3}$ atoms/s, implying 3-4 orders improvement compared to MIT work. Optical diagnostics via the $1 \mathrm{~S}-2 \mathrm{~S}$ two-photon transition [24, 25] is another option, which would be certainly beneficial and would allow verifying many properties of ultra-cold $\mathrm{H}$ gas.

\section{Conclusions}

We suggested two configurations of experiments with ultra-slow atoms of atomic hydrogen with the energies comparable with their gravitational potential on the height of a fraction of $1 \mathrm{~mm}$. At the first stage of this project, studies of quantum reflection of these atoms from the surface of superfluid helium will allow further insights into the physics of interactions of atoms with surfaces in the ultra-low energy regime and quantitative measurements of van der Waals/Casimir-Polder forces. This information is of major importance for designing similar experiments with anti-hydrogen atoms currently pursued in CERN.

Acknowledgements Open access funding provided by University of Turku (UTU) including Turku University Central Hospital. This work was supported by the Wihuri Foundation and grant of the Academy of Finland 317141. The authors are grateful to colleagues from GRANIT and GBAR collaborations for useful and stimulating discussions.

Open Access This article is distributed under the terms of the Creative Commons Attribution 4.0 International License (http://creativecommons.org/licenses/by/4.0/), which permits unrestricted use, distribution, and reproduction in any medium, provided you give appropriate credit to the original author(s) and the source, provide a link to the Creative Commons license, and indicate if changes were made.

Publisher's note Springer Nature remains neutral with regard to jurisdictional claims in published maps and institutional affiliations.

\section{References}

1. Nesvizhevsky, V.V. et al.: Quantum states of neutrons in the Earth's gravitational field. Nature 415, 297 (2002)

2. Fried, D.G. et al.: Bose-Einstein condensation of atomic hydrogen. Phys. Rev. Lett. 81, 3811 (1998)

3. Anderson, M.H., Ensher, J.R., Matthews, M.R., Wieman, C.E., Cornell, E.A.: Observation of BoseEinstein condensation in a dilute atomic vapor. Science 269, 198 (1995)

4. Davis, K.B., Mewes, M.O., Andrews, M.R., Van Druten, N.J., Durfee, D.S., Kurn, D.M., Ketterle, W.: Bose-Einstein condensation in a gas of sodium atoms. Phys. Rev. Lett. 75, 3969 (1995)

5. Safonov, A. et al.: Observation of quasicondensate in two-dimensional atomic hydrogen. Phys. Rev. Lett. 81, $4545.4548(1998)$

6. Voronin, A.Y., Froelich, P., Nesvizhevsky, V.V.: Gravitational quantum states of antihydrogen. Phys. Rev. A 83, 032903 (2011)

7. Kellerbauer, A. et al.: Proposed antimatter gravity measurement with an antihydrogen beam. Nucl. Instr. Meth. B. 266, 351 (2008)

8. Charman, A. et al.: Description and first application of a new technique to measure the gravitational mass of antihydrogen. Nature Commun. 4, 1785 (2013)

9. Indelicato, P. et al.: The Gbar project, or how does antimatter fall? Hyperf. Int. 228, 141 (2014)

10. Pérez, P. et al.: The GBAR antimatter gravity experiment. Hyperf. Int. 233, 21 (2015)

11. Silvera, I.F., Walraven, J.T.M.: Stabilization of atomic hydrogen at low temperature. Phys. Rev. Lett. 44, 164 (1980)

12. Hess, H.H.: Evaporative cooling of magnetically trapped and compressed spin-polarized hydrogen. Phys. Rev. B 34, 3476 (1986)

13. Crepin, P.P. et al.: Quantum reflection of antihydrogen from a liquid helium film. Europ. Phys. Lett. 119, 33001 (2017) 
14. Berkhout, J.J. et al.: Vanishing sticking probabilities and enhanced capillary flow of spin-polarized hydrogen. Phys. Rev. Lett. 57, 2387 (1986)

15. Berkhout, J.J. et al.: Quantum reflection: focusing of hydrogen atoms with a concave mirror. Phys. Rev. Lett. 63, 1689 (1989)

16. Doyle, J.M. et al.: Hydrogen in the submillikelvin regime: Sticking probability on superfluid 4 He. Phys. Rev. Lett. 67, 603 (1991)

17. Pinkse, P.W.H., Mosk, A., Weidemuller, M., Reynolds, M.W., Hijmans, T.W., Walraven, J.T.M.: Onedimensional evaporative cooling of magnetically trapped atomic hydrogen. Phys. Rev. A 57, 4747 (1998)

18. Fried, D.G.: Bose-Einstein Condensation of Atomic Hydrogen. Ph.D. thesis MIT (1999)

19. Doyle, J.M.: Energy Distribution Measurements of Magnetically Trapped Spin Polarized Atomic Hydrogen: Evaporative Cooling and Surface Sticking. Ph.D. thesis MIT (1991)

20. Attocube systems AG. ANPz101. www.attocube.com

21. Ichikawa, G. et al.: Observation of the Spatial Distribution of Gravitationally Bound Quantum States of Ultracold Neutrons and its Derivation Using the Wigner Function. Phys. Rev. Lett. 112, 071101 (2014)

22. Nesvizhevsky, V.: ILL Internal Report 96NE14T, p. 1 (1996)

23. Cabrera, B. et al.: Detection of single infrared, optical, and ultraviolet photons using superconducting transition edge sensors. Apl. Phys. Lett. 73, 735 (1998)

24. Huber, A., Gross, B., Weitz, M., Hänsch, T.W.: High-resolution spectroscopy of the 1S2S transition in atomic hydrogen. Phys. Rev. A 59, 1844 (1999)

25. Killian, T.C.: 1S-2s Spectroscopy of Trapped Hydrogen: the Cold Collision Frequency Shift and Studies of BEC. Ph.D. thesis MIT (1999) 